\title{
A 3-D hydrodynamical simulation of wind accretion
}

\author{
Henri M.J. Boffin \\ Institut d'Astronomie et d'Astrophysique \\ C.P. 165, Université Libre de Bruxelles \\ 50, av. F.D. Roosevelt \\ B-1050 BRUSSELS
}

\begin{abstract}
Using a 3-dimensional hydrodynamical code, based on the Smooth Particle Hydrodynamics (SPH) method, we have simulated the case of wind accretion. Several configurations were studied:

- homogeneous mass flow

- wind with a density gradient

- wind with a velocity gradient

In each case, the accretion rate as well as the accreted angular momentum has been calculated. Comparisons were done with other simulations published in the literature and especially with the two currently existing 3-D simulations. According to one of these group (Matsuda et al., 1990) resolution is very critical in determining the accretion rate as well as to determine if the flow is stationary or not. We have done simulation at various resolutions and we confirm its importance for the deduced accretion rate. However, our simulations do not seem to show any non- stationary behaviour.
\end{abstract}

\section{Introduction}

In a binary system, mass transfer can occur in 2 different ways not mutually exclusive: if the system is close enough, Roche lobe overflow will arise (and will operate in most cases on thermal or even dynamical timescales). The other possibility of mass transfer is wind accretion (which occurs on a nuclear timescale). Most of the stars (especially hot stars or late-type giants) can lose an appreciable quantity of mass by wind. As this wind is more or less ejected isotropically, the orbiting companion can intercept part of this mass, and therefore undergo changes in its chemical composition or even more drastic changes. Wind accretion in a binary system could be the cause of as many different situations as Barium stars (see Boffin \& Jorissen, 1988), Symbiotic stars, novae, SNIa, X-ray binaries,etc...

The understanding of the wind accretion process is therefore critical to get the clue to the origin of those peculiar systems.

\section{The basic problem}

Let us first consider the basic problem: matter is flowing from infinity with a velocity $\mathrm{v}_{\infty}$ and a density $\rho_{\infty}$ towards a gravitational point source. Far away from the object, forces caused by pressure gradients and viscosity will be small and the matter will follow a free Keplerian orbit. Behind the object, there will be a region (either an 'accretion-column' or an 'accretion cone') where particles collide and increase the density. In this region, pressure effects will become important.

The first attempt to solve this was done by Hoyle \& Lyttleton (1939) who derived an accretion rate:

$$
\dot{\mathrm{M}}_{\mathrm{HL}}=\pi \rho_{\infty} \mathrm{v}_{\infty} \mathrm{R}_{\mathrm{HL}}^{2}
$$

where $R_{H L}$ is the accretion radius given by

$$
\mathrm{R}_{\mathrm{HL}}=\frac{2 \mathrm{GM}}{\mathrm{v}_{\infty}^{2}}
$$


$\mathrm{G}$ being the gravitational constant and $\mathrm{M}$ the mass of the gravitating object. This result was obtained by neglecting all pressure effects and assuming purely Keplerian trajectories for the particles. Thus, it is only interesting for the case of an hypersonic flow. Bondi \& Hoyle (1944), by a more realistic approach, including some limited pressure effects along the 'accretion-column', showed that Eq.(1) was an upper limit and that the actual accretion rate is between 0.5 and 1 times this value. Later, Bondi (1952) studied the complementary case (only pressure effects) of a stationary spherically symmetric accretion with $\mathrm{v}_{\infty}=0$. In this case, he obtained a result similar to Eq.(1), the (maximum !) accretion radius being this time

$$
\mathrm{R}_{\mathrm{B}}=2 \frac{\mathrm{GM}}{\mathrm{c}_{\infty}^{2}}
$$

where $c_{\infty}$ is the sound speed at infinity. He proposed that the accretion rate in the intermediate case could be given by the following formula:

$$
\dot{\mathrm{M}}_{\mathrm{BH}}=\alpha \dot{\mathrm{M}}_{\mathrm{HL}} \frac{\mathrm{v}_{\infty}^{3}}{\left(\mathrm{v}_{\infty}^{2}+\mathrm{c}_{\infty}^{2}\right)^{1.5}}
$$

where $0.5<\alpha<1$. One has to bear in mind that this relation has no physical justification but is just a guess for an interpolation formula.

In view of the complexity of the problem and the difficulty to solve it analytically, numerical simulations were called for help. Most of them are 2-D and seem to give reasonable agreement with Eq.(1) or (2) (see e.g. Hunt 1971, de Kool \& Savonije 1988,...). However, in order to extend these results to the real situation of a binary system (i.e. incorporating isotropic ejection and pseudo-forces), 3-D calculations should be used. Until now, only two 3-dimensional simulations exist. The first one (Livio et al. 1986) concludes that, for a given Mach number, $\alpha$ is a function of the polytropic index, $\gamma$. This simulation has however some problems with the grid size so that the results with $\gamma=1.5$ and $\gamma=5 / 3$ should be taken with some caution.

The second 3-D simulation (Sawada et al. 1989, Matsuda et al. 1990) has shown several results:

i) the details of the accretion flow are different in the $3 \mathrm{D}$ and $2 \mathrm{D}$ cases.

ii) the resolution is crucial for the result:

a) with lower resolution, the flow is steady, while at higher resolution, it becomes non-steady.

b) the accretion rate is also function of the resolution.

- in 2D: $\dot{\mathrm{M}} \simeq(.75$ to 1$) \dot{\mathrm{M}}_{\mathrm{BH}}^{\max }$ for $\mathrm{v}_{\infty}=3 \mathrm{c}_{\infty}$, depending on the resolution and on $\gamma$. However, for $\mathrm{v}_{\infty}=5 \mathrm{c}_{\infty}, \dot{\mathrm{M}} \simeq 0.5 \dot{\mathrm{M}}_{\mathrm{BH}}^{\mathrm{max}}$ size.

- in $3 \mathrm{D}: \dot{\mathrm{M}} \simeq(.3$ to .8$) \dot{\mathrm{M}}_{\mathrm{BH}}^{\max }$ depending on the number of cells and on the accreting body

The situation seems thus very complicated and deserves further study.

We have therefore undertaken a 3-D numerical simulation of wind accretion using the smooth particle method (SPH).

\section{Numerical simulations}

We have done numerical simulations of the basic problem with $\mathrm{M}_{\infty}\left(\equiv \mathrm{v}_{\infty} / \mathrm{c}_{\infty}\right)=2.33$ and $\gamma=4 / 3,3 / 2$ and 5/3. In this case, $\mathrm{R}_{\mathrm{HL}}=.37$ and $\dot{\mathrm{M}}_{\mathrm{HL}}=\alpha$, while $\dot{\mathrm{M}}_{\mathrm{BH}}=.77 \alpha$. For $\gamma=5 / 3$, we have considered 2 resolutions: $h=0.08$ and $h=0.04$. In those units, the resolution of Matsuda et al. (1990) was 0.027 and 0.054 . A plot of the flow obtained is shown on figure 1 . For the $h=0.08$ case (low resolution), about 8500 particles were used and we needed about 2 CPU hours on a Cray XMP. For $h=0.04$, we used about 73000 particles and 60 CPU hours. This is about the maximum allowed by the memory of the Cray XMP.

In each case, the flow appeared to be stationary and the accretion rate obtained was, for $\gamma=5 / 3$, $\dot{\mathrm{M}}=.85$ for $\mathrm{h}=0.08$ and $\dot{\mathrm{M}}=.67$ for $\mathrm{h}=0.04$. It therefore appears that the accretion rate is in our case also depending on the resolution used. 


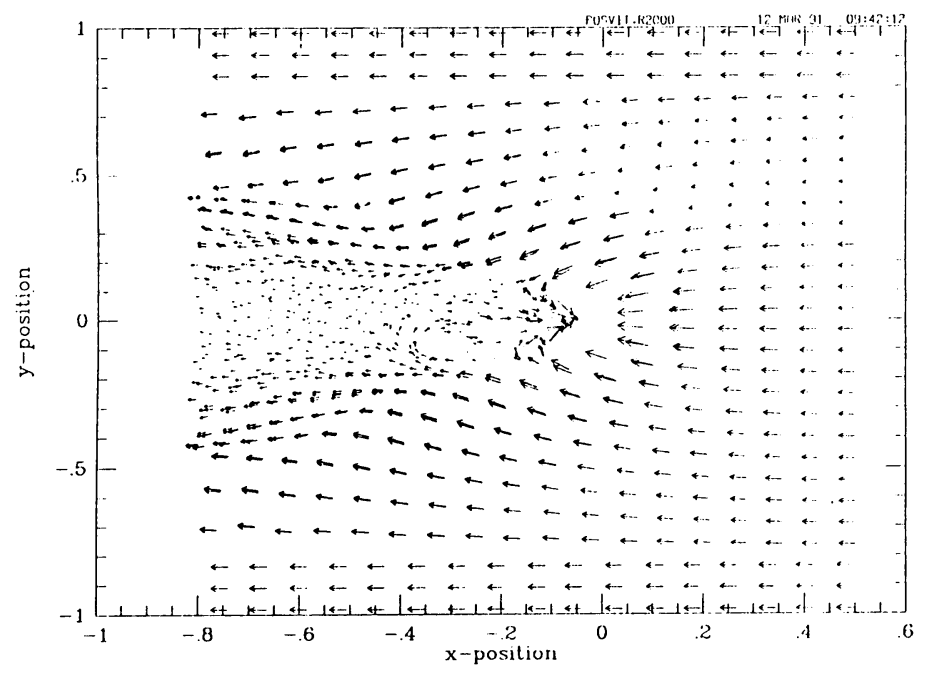

Figure 1: Velocity field in the $z=0$ plane for the homogeneous case with $\mathrm{M}_{\infty}=2.33$, $\gamma=5 / 3, h=0.08$.

\section{Density and velocity gradient}

If we want to consider the case of accretion in a binary system, then the study of a homogeneous cloud is maybe too far from reality. The next step which is usually done is to study the case of a density or a velocity gradient in the cloud. If we do not expect that this will change the accretion rate, we can hope that this will bring valuable informations about the angular momentum accreted.

Let us consider a flow which at infinity has either a gradient in density (i.e. $\rho_{\infty}=\rho_{\mathrm{o}}(1+\epsilon \mathrm{y}) ; \mathrm{v}_{\infty}=$ $\left.v_{o}\right)$, or either a gradient in velocity (i.e. $\left.v_{\infty}=v_{o}(1+\epsilon y) ; \rho_{\infty}=\rho_{o}\right)$. If one assumes that all the angular momentum which is inside the accretion cylinder with radius $\mathbf{R}_{\mathrm{a}}$ (accretion radius) is accreted, then one gets:

$$
\begin{array}{ll}
\dot{J}_{B H}=4 \pi \epsilon \frac{\rho_{o}}{M_{\infty}^{6}} & \text { density gradient } \\
\dot{J}_{B H}=-24 \pi \epsilon \frac{\rho_{o}}{M_{\infty}^{6}} & \text { velocity gradient } .
\end{array}
$$

Numerical simulations by Livio et al. (1986) and Sawada et al. (1989) have shown that the actual accreted angular momentum is much smaller than those conservative values.

We have performed numerical simulations with $h=0.08$ for the case of a density gradient as well as for a velocity gradient, using always $\epsilon=0.3$. An instantaneous picture of the flow in the case of a density gradient is shown in figure 2 . The accretion rates were in both cases equal to $\dot{M}=0.9$ and thus not very far from the value obtained for the homogeneous case.

The accreted angular momentum was:

i) $|\dot{J}|<10^{-3} \quad\left(\right.$ i.e. $\left.<\dot{J}_{B H} / 20\right)$ density gradient

ii) $|\dot{J}|=0.018 \quad\left(\right.$ i.e. $\left.=\dot{J}_{B H} / 8\right)$ velocity gradient 


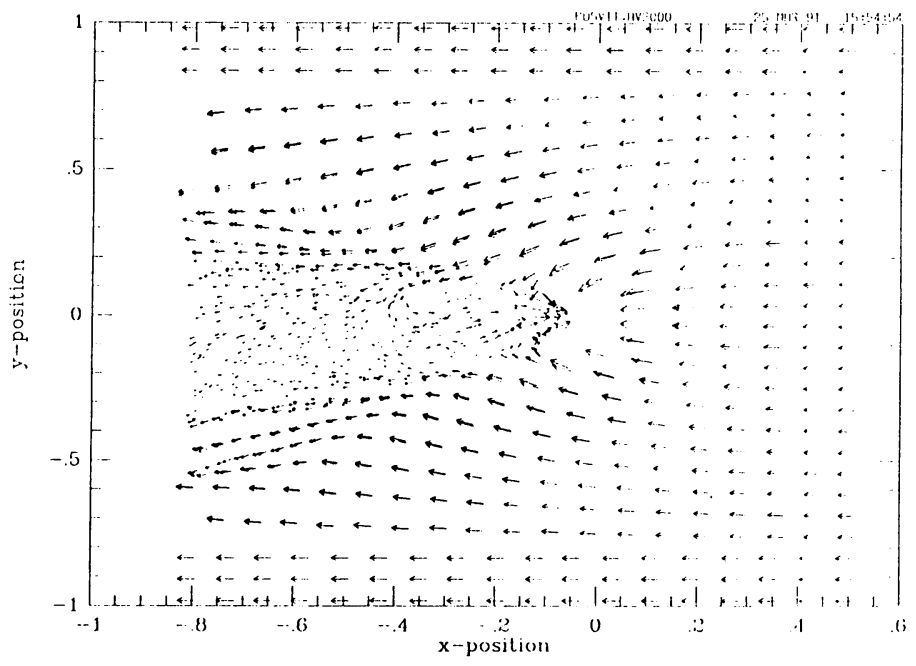

Figure 2: Velocity field in the $z=0$ plane for the case of a density gradient with $\mathrm{M}_{\infty}=2.33$, $\gamma=5 / 3, h=0.08, \epsilon=0.3$.

\section{Conclusion}

We have realized 3D numerical simulations of wind accretion using the SPH method. In the homogeneous case, the accretion rate obtained was in fair agreement with the Bondi-Hoyle value, but seems to be dependent on the resolution. We do not get the non-steady behaviour obtained by Matsuda et al. (1990), but this could be due to the fact that our resolution is still not high enough and that numerical viscosity is still too important. We must emphasize however that our high resolution simulation was the maximum allowed by our code using a Cray XMP of 4 MW of memory. Adding the fact that this simulation was very costly in CPU hours, it can be said that it will be very difficult to get a higher resolution with the SPH method using our current computation resources. For the case with a density or a velocity gradient, the accretion rate is not modified, but the accreted angular momentum is much smaller than the conservative value of $\dot{J}_{B H}$.

Acknowledgements Part of this work has been done while the author was guest of the Max-Planck Institute für Astrophysik (Garching bei München). It is a pleasure to thank Prof. W. Hillebrandt for his kind hospitality. The author is grateful to N. Cerf for useful discussions. Calculations were made in part on the Cray XMP of MPA and in part on the Cray XMP of the Université Libre de Bruxelles.

\section{References}

Boffin H.M.J., Jorissen A.: 1988, Astron. Astrophys. 205, 155.

Bondi H.: 1952, Mon. Not.R.astr.Soc 114,195

Bondi H., Hoyle F.: 1944, Mon. Not.R.astr.Soc 104,273

de Kool M., Savonije G.J.: 1988, Astron. Astrophys. 189,331

Hoyle F., Lyttleton R.A.: 1939, Proc. Camb. Phil. Soc. $\underline{35}, 405$.

Hunt R.: 1971, Mon. Not.R.astr.Soc. 154, 141

Livio M., Soker N., de Kool M., Savonije G.J.: 1986, Mon. Not.R.astr.Soc. 222,235

Matsuda T., Sekino N., Sawada K., Shima E., Livio M., Anzer U., Börner G.: 1990, preprint MPA.

Sawada K., Matsuda T., Anzer U., Börner G., Livio M.: 1989, Astron.Astrophys. 221,263 